\title{
Precise Distributed Localization Algorithms for Wireless Networks
}

\author{
Saad Biaz and Yiming Ji \\ Computer Science and Software Engineering Department \\ Auburn University \\ 107 Dunstan Hall, Auburn University, AL, 36849-5347, USA \\ \{jiyimin,sbiaz\}@auburn.edu \\ Technical Report CSSE05-02 (March 4, 2005)
}

\begin{abstract}
We propose in this paper reliable and precise distributed localization algorithms for wireless networks: iterative multidimensional scaling (IT-MDS) and simulated annealing multidimensional scaling (SA-MDS). We use only radio communication constraints to infer node's distances, and uniquely combine multidimensional scaling algorithm (MDS) with lateration algorithm. We analytically establish the upperbound on the estimation error. The proposed techniques can estimate all nodes' positions even with limited and imprecise network knowledge. Analysis and test runs show that the proposed methods are independent from the topology randomness and the range measurement errors. We attempt to bound the localization imprecision. Simulation results yield an average estimation error of about $25 \%$ of radio transmission range. And the highest estimation error observed is $40 \%$ radio range for most networks.
\end{abstract}

\section{Introduction}

In wireless ad hoc networks, location information can be useful for coverage analysis [1], mobility [2], environmental monitoring [3], target tracking [4], battle-field surveillance [5], intrusion detection [6], and location-aware applications such as location-aided routing $[7,8]$.

The location can be obtained by GPS enabled systems with a good precision. However, GPS system cannot be used for in-door applications and is inappropriate for large scale deployment of ad hoc sensor networks because of constraints in volume, power consumption and cost.

An alternative to GPS is the use of distributed localization algorithms that enable nodes to determine their relative positions. If absolute positions are necessary, a limited number of nodes (called anchors) must have the capability of determining their absolute positions. Anchors can get their absolute position using manual configuration or techniques such as GPS. Much localization research has been carried out recently using this approach $[9,10,11,12,13]$. Despite real advances in localization techniques, three problems remain with existing algorithms:

1. Most algorithms work only under specific and restrictive network conditions. When such conditions are not met, the confidence in location estimates is not satisfactory and makes key decisions hard and risky;

2. Most algorithms require excessive network communications and complex refinement procedures with multiple computing rounds that are energy inefficient for small devices in ad hoc networks;

3. Precision evaluation of these algorithms is conducted using different metrics and criteria making uneasy the comparison of algorithms.

In this paper, we attempt to address these problems. First we define the performance evaluation method that can be used to evaluate and compare all localization methods. Second, we propose a technique that can: i) depend less on the hardware support, ii) yield accurate location estimates even with imprecise inputs, iii) work for general networks with a predictable precision, and iv) be computation and communication efficient.

We consider the radio communication constraints [14] to infer raw estimates of the position of a node or the distance between nodes. We mean by radio communication constraints the set of geometry rules among the communicating nodes. The constraints can be radial and angular restrictions or a combination of them that are used to bound the position estimates. For example, if one node $(A)$ can receive message from another node $(B)$, then the distance between these two nodes should be less than node $B$ 's radio transmission range.

We propose two methods that are built upon two efficient algorithms: lateration algorithm [15, 9] and the multidimen- 
sional scaling algorithm (MDS) [16, 17]. We adapt these algorithms to work and yield accurate estimates even with imprecise and/or incomplete information. Different from X. Ji's method in [11], our methods are practical in that we specially take into consideration imprecision the radio propagation technique, and we use an estimated n-hop $(n>1)$ pair-wise distance as inputs.

Through simulation, we establish that IT-MDS and SAMDS methods provide better location estimates than pure MDS or pure lateration, no matter of the network deployment randomness, the network density, and 1-hop range measurement errors. For a unit square-area network with more than $10 \%$ anchors (nodes with known absolute positions), and a radio transmission range of 0.2 , the average prediction error for both algorithms is bounded by $40 \%$ radio range. Also, if we use $D V$-Hop propagation method [15] (see section 3.1), both IT-MDS and SA-MDS algorithms are robust to the 1-hop pair-wise distance measurement errors. If Euclidean propagation method [15] is used, both algorithms provide precise estimates.

The rest of the paper is organized as follows: In Section 2, we present the research settings and definitions, and introduce the radio communication constraint models. In Section 3, we briefly describe the localization algorithms shown in recent literature. Section 4 will discuss our localization approach, and Section 5 will introduce the experiment environment and the test results. Section 6 will conclude the paper and point future work.

\section{Definitions and Assumptions}

We consider a static ad hoc wireless network in a unit square area. We assume that all nodes transmit radio with the same maximum range $R(0 \leq R \leq 1)$, and that they can measure the distance to their directly connected neighbors. In addition, we assume the network contains a small set of anchor nodes with prior knowledge of their positions (the others are called normal nodes). If there are $m$ anchors for an ad hoc network with total $N$ nodes, the anchor density is defined as $\frac{m}{N}$.

\subsection{Deployment Randomness}

For a network, if all nodes strictly reside on crosspoints of a coordinate grid then the deployment randomness is null. Specifically, the deployment randomness is defined as the maximum displacement $x$ of each node that can swing from a crosspoint. Starting from a null deployment, each node is displaced from a crosspoint over a random distance in a random direction. The unit of the maximum displacement is the radio transmission range. We associate a random number $\rho_{i}(\phi \in[0,1])$ with each node $n_{i}$. For example, if the randomness is $x$, then the maximum displacement is $x \cdot R$, and the displacement of node $n_{i}$ is $\phi_{i} \cdot x \cdot R$. Similarly, a random number $\theta_{i}$ is assigned for the direction of the displacement.

\subsection{Estimation Error}

For a set of nodes, we denote the estimation error as the average distance between true positions and the estimated positions. The estimation error is expressed as a fraction of the radio range. Let $X_{i}(i=1,2, \ldots, n)$ be the coordinates of $n$ normal nodes where $X_{i}=\left(x_{i 1}, x_{i 2}, x_{i 3}\right)^{T}$. If $\widehat{X}_{i}$ are the estimated coordinates, then the estimation error is given by the euclidean distance between $X_{i}$ and $\widehat{X_{i}}$ :

$$
e r r=\frac{\sum_{i=1}^{n} \sqrt{\left(X_{i}-\widehat{X_{i}}\right)^{T}\left(X_{i}-\widehat{X}_{i}\right)}}{n \cdot R}
$$

\subsection{Fitness Function}

Consider a path with $n$ nodes that measure their pair-wise distances. The measured pair-wise distance of all nodes over this path is denoted by $\Delta=\left[\delta_{i, j}, i, j=1, \ldots, n ; i<j\right]$, where $\delta_{i, j}=\left(X_{i}-X_{j}\right)^{T}\left(X_{i}-X_{j}\right)$. Based on these measurements, a localization algorithm provides estimates of the positions of the nodes. If the pair-wise distance between the estimates is given by $\widehat{\Delta}$, with $\widehat{\Delta}=\left[\widehat{\delta_{i, j}}, i, j=\right.$ $1, \ldots, n] ; i<j)$ and $\widehat{\delta_{i, j}}=\left(\widehat{X_{i}}-\widehat{X_{j}}\right)^{T}\left(\widehat{X_{i}}-\widehat{X_{j}}\right)$.

The fitness function is defined as the difference between $\widehat{\Delta}$ and $\Delta$, and it is denoted by $\sigma$, where:

$$
\sigma=\sum_{i<j} \frac{\left(\widehat{\delta_{i j}}-\delta_{i j}\right)^{2}}{\delta_{i j}^{2}}
$$

The goal of the localization algorithms is to minimize the fitness function, and to obtain minimum estimation error.

\subsection{Radio Communication Constraints}

Radio communication constraints are a set of geometry rules useful to bound position estimates. Generally, radio communication constraints are a combination of radial and angular constraints [14].

Radial constraint means that if a node $B$ can hear a node $A$, the distance between them is less than $A$ 's radio transmission range. In Figure 1(a), since nodes $B_{1}$ and $C_{1}$ hear node $A_{1}$, then the distance of $A_{1} B_{1}$ and $A_{1} C_{1}$ is less than $A_{1}$ 's radio range $R$. In Figure 1 (b), assume that node $A_{2}$ can transmit with two different ranges: $R_{1}$ and $R_{2}$ $\left(R_{1}>R_{2}\right)$. If node $B_{2}$ hears node $A_{2}$ only when $A_{2}$ uses its highest power with range $R_{1}$, then $B_{2}$ is in the circular ring-belt between $R_{1}$ and $R_{2}$.

Angular constraint refers to the fact that when a node gets the best reception at certain angle, it can estimate the 


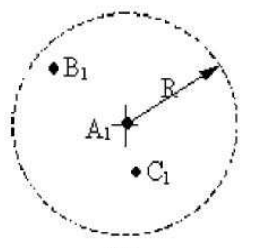

(a)

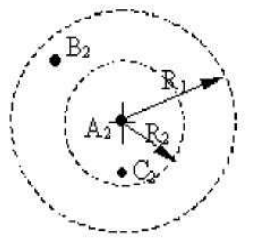

(b)

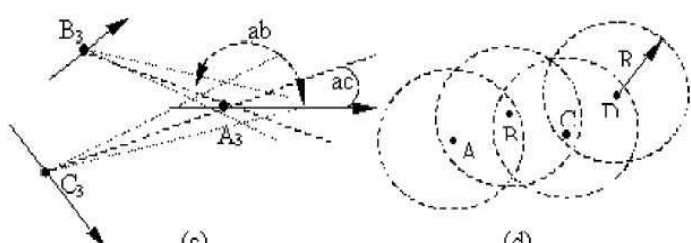

(c)
Figure 1. (a) Radio with fixed transmission range. (b) Radio with various ranges. (c) Angular constraint. (d) N-hop neighborhood constraint

relative angle to the source transmitter, which may be a cone bounded by certain limit [14]. In Figure 1 (c), the location of node $A_{3}$ can be determined with relative angles of $a c$ and $a b$.

In addition, a small group of neighbors may form a set of triangles with local geometry constraints to further precise estimates [18]. In Figure 1(d), nodes $A, B, C$ and $D$ are neighbors along a path from $A$ to $D$. The distance between $A$ and $D$ is less than $3 R$. The distance between $A$ and $C$, as well as the distance between $B$ and $D$ should be less than $2 R$. In addition, $B$ resides in the radio transmission intersection area of $A$ and $C$, and node $C$ is within the intersection area of $B$ and $D$.

\section{Related localization algorithms}

\subsection{Radio Propagation Method}

A localization procedure starts with anchor nodes broadcasting their position information to the whole network. Upon receiving an anchor's packet, a normal node will measure the distance or angle to the neighboring sender (onehop pair-wise distance). Then it may rebroadcast the packets to other neighbors after updating the hop count on the received anchor packet. So far, nodes can estimate their position to anchors only in terms of hop count.

In order to infer from the radio constraints the physical distances between nodes, several mechanisms have been proposed and somewhat relate to the following four methods:
- DV-Hop propagation method [15, 19]: In this method, each node maintains, updates, and rebroadcasts only packets with the smallest number of hop counts. Each packet bears a hop count field that is updated each time the packet is forwarded. Starting from an anchor node, hop by hop information propagation allows a node to determine its distance, in hop units, to that anchor. The physical distance can be deduced using an average hop distance.

- DV-Distance method $[15,20]$ : This method is similar to $D V$-Hop, except that each node now attempts to measure the real pair-wise distance between neighboring nodes. The hop by hop information propagation transmits the cumulative real distance instead of hop counts.

- Euclidean method [15]: This method allows each node to calculate the distance to the anchor using the local geometry algorithms based on the range estimation between neighboring nodes. This method is complex, and may induce cumulative errors if the radio range measurement is not precise.

- Kleinrock's formula [21]: Kleinrock and Silvester proposed a formula to compute the average hop distance by using the idea of local neighborhood density $N_{\text {local }}=n \frac{\pi R^{2}}{\mathcal{A}}$ where $n$ is the total number of nodes in the network, $R$ is the radio transmission range, and $\mathcal{A}$ is the total area where the network is deployed. Kelinrock and Silvester express the average hop distance $d_{\text {hop }}$ as:

$d_{\text {hop }}=R\left(1+e^{-N_{\text {local }}}-\int_{-1}^{1} e^{-\frac{N_{\text {local }}\left(\arccos t-t \sqrt{1-t^{2}}\right.}{\pi}} d t\right)$

Nagpal et al. [22, 23] used this formula in the Amorphous localization algorithm, and found that the value of 15 for $N_{\text {local }}$ is a critical minimal threshold for achieving low errors in the distance estimation.

DV-Hop and DV-Distance methods are simple and do not require dense deployment. The hop counts or the distances the nodes maintained are actually the minimum records along a shortest path to the anchors. On the contrary, the Euclidean and Kleinrock methods only work in high density networks. And Euclidean method require complex calculations.

In our study, we used the DV-Hop method to forward anchor's packets, and further to estimate the distance to anchors. For comparison, we also used the Euclidean method (with various accumulated errors) on the proposed algorithms. 


\subsection{Localization Algorithms}

Based on their specific estimation approach, localization algorithms fall into one of these categories:

1) Lateration: given distance measurements from a node to 3 or more anchors, a system of equations is derived and then solved using least squares method [15, 24];

2) Bounding-box: given a set of neighbors' or anchors' position advertisements, a node derives a probable region in which it is most likely located [9, 10, 14]. The center of gravity of the intersection is picked as the final estimate.

3) Multidimensional Scaling method: a traditional social analysis technique to derive relative relations among objects. If Euclidean distance is considered as dissimilarities between object points, relative coordinates can be determined [17].

4) Grid overlaying method: an overlapping method similar to bounding-box algorithm. However, it denotes a node's possible position as a set of grid coordinates. The local area is updated iteratively through communication, and the final estimate is the average of the current set of grid coordinates $[12,25]$.

5) Probabilistic method was proposed by Ramadurai et al. in [26, 27]. The key element of this method is the probability distribution function of the radio transmission strength. If a normal node is reached by multiple anchor radios, the overlapping space with highest probability is selected as the estimated position.

The bounding-box method, probabilistic method and grid overlaying method are similar as they all are based on the intersection mechanism to obtain the possible final area. Lateration method and multidimensional method are classical methods. Lateration uses least squares algorithm to calculate each node's position individually. Differently, MDS uses matrix decomposition and it can estimate the positions for a set of nodes along a radio propagation path in one processing round. Therefore, compared with the lateration method, MDS algorithm is more computation efficient.

In this paper, we will present two MDS based algorithms, iterative MDS (IT-MDS) and and simulated annealing MDS (SA-MDS). In [11], X. Ji et al proposed a similar iterative method in their research. The detailed approach of the two iterative algorithms is different. Specially, the two methods differ as follows:

1. Estimation coverage: For a general network without special deployment, simple MDS based algorithm can not provide full coverage estimation for all nodes in the network. We further detail this issue in section 4.3;

2. Constraints: We specially consider the radio communication constraints during each iteration loop;

3. Fitness function: We use adapted fitness function without considering all pair-wise distance;
4. Mechanism: Our localization procedure is transparent to end users in that we specifically combine the relative position information with the anchors' physical position, this way, local map is NOT necessary to be constructed at all individual normal nodes. This mechanism is more energy efficient;

5. Performance: X. Ji's iterative algorithm doesn't always provide optimal estimates (section 5.3).

In the following sections, We first present the iterative MDS (IT-MDS) algorithm, then we describe the simulated annealing based MDS (SA-MDS) algorithm. In section 5.3, we compare the proposed algorithms with other representative localization algorithms.

\section{Augmented multidimensional scaling}

Our localization method is a combination approach of the multidimensional scaling method and the lateration method. Basically, the algorithm works as follows:

1) Anchors start the estimation process by broadcasting their position information to the whole network. Using the DV-Hop or Euclidean radio propagation method, each intermediate node maintains the minimum hop distance to the anchors.

2) If a path $\mathcal{P}$ contains more than three anchors, the information collected through $\mathcal{P}$ is transmitted back to the original anchors for localization procedure.

3) The original anchor processes the path such that the path ends with another anchor node. The average hop size is calculated by averaging the physical distance between ending anchors with the hop count between them.

4) Apply IT-MDS or SA-MDS algorithm for the nodes along the paths, and update the average hop size iteratively. (The average hop size will be used by lateration algorithm).

5) Apply the lateration algorithm for the rest nodes (not on paths with three anchors).

6) Possibly refines the positions using the constraints from neighbors

\subsection{Classical and iterative MDS}

Classical multidimensional scaling (MDS) is a metric MDS technique in 1930s by Young and Housholder [16]. It is widely used in many areas including social science, and there are many software package are available. A good reference for MDS is by Cox in [17].

To apply the MDS algorithm in the estimation of the nodes' position in a wireless network, accurate pair-wise distance among all nodes along transmission paths is needed. If absolute position is required, each path must contain at least three anchor nodes in order to adjust the relative 
position accordingly. However, detail pair-wise distance information is not available in most cases. In reality, only the following information along a path is acheavable: 1) The anchors' positions; 2) 1-hop pair-wise distance; 3) Estimated average hop size; and 4) Hop counts between pairs.

Therefore, iterative approach is proposed to optimize the estimation with the incomplete range information. With limited pair-wise distance information, we modify the fitness function $\sigma$ (equation 2) accordingly. Specifically, we assume the pair-wise distance matrix $\Delta$ and $\widehat{\Delta}$ include only the 1-hop pair-wise distance between nodes in the path, where $\Delta$ represents the measured 1-hop pair-wise distance and $\widehat{\delta}$ denotes the estimates for each iteration. The reason is that the measured 1-hop distance is generally more precise than the estimated n-hop $(n>1)$ pair-wise distance.

Based on the neighborhood radio communication constraints and the accurate anchors position information, the algorithm will minimize the pair-wise distance errors iteratively. At each iteration round, if the new fitness function is less than the previous, the position estimation updates will be kept until specific requirement is fulfilled. Therefore, the main procedure can be described as follows:

while $(\sigma>\epsilon)\{$

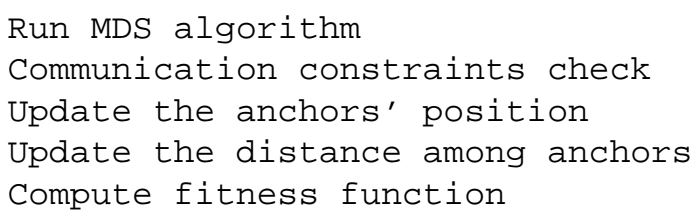

The communication constraints check is used to bound the position updates after each MDS process within the iteration loop. Based on the imprecise $\mathrm{n}(n>1)$ hop pair-wise distance from hop count, MDS algorithm is likely to yield unrealistic position estimates, therefore, it is necessary to bound or reposition those estimates according to the neighborhood radio communications.

Figure 2 gives an example for the IT-MDS algorithm. The left figure shows the converge procedure of the IT-MDS algorithm: the '+' presents the true position of the nodes along one path; 'o' presents intermediate estimates. The anchors are denoted by $\otimes$, and they are fixed during the iteration. The right figure shows the fitness function corresponding to the iteration progress.

The IT-MDS algorithm works well when the anchor nodes are not aligned. In our implementation, we use the following methods:

i) if there are more than three anchors in the path, we can select a reference anchor with the longest distance to the line formed by the starting and the ending nodes;

ii) if all anchors are on the same line, neglect the estimation procedure;
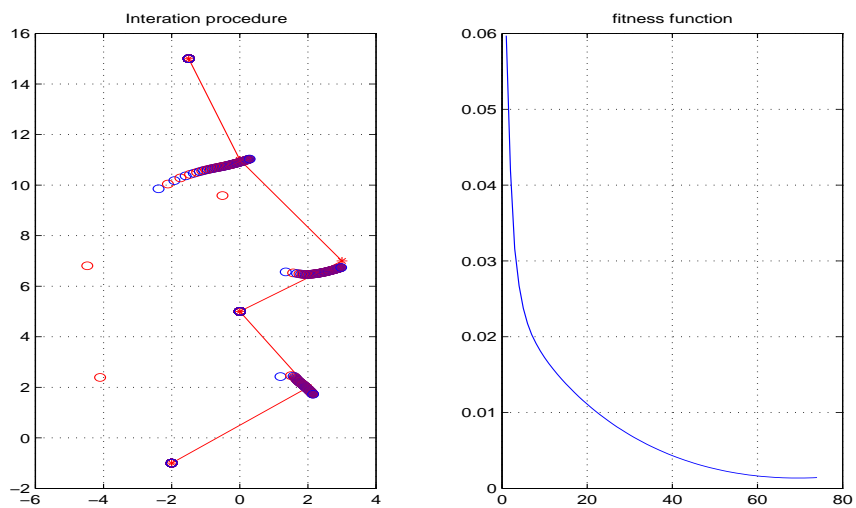

Figure 2. Iterative MDS algorithm example

iii) if one node can reside on multiple routes and get estimates more than once, the final position estimates for that node is given as the average of all predictions.

\subsection{Simulated Annealing MDS Algorithm}

Simulated Annealing (SA) [28, 29] is a method used to search for a minimum in a general system. It is based on the process of the way a metal cooling down to the optimal state (the annealing process). SA's major advantage is an ability of a random search which not only accepts changes that decrease objective function, but also some changes that increase it. Thus, SA method can achieve global optimization without becoming trapped at local minima.

The connection between SA algorithm and mathematical minimization was first intorduced by Pincus [30], later, Kirkpatrick et al. [31] provided the basis of an optimization technique for combinatorial (and other) problems.

The original Metropolis scheme [28] indicated that an initial state of a thermodynamic system was chosen at energy $\mathrm{E}$ and a desired temperature T. Holding at that temperature $\mathrm{T}$, the initial configuration is perturbed and the change in energy $\mathrm{dE}$ is computed. Applying Monte Carlo sampling techniques, the physical annealing process has been modeled successfully by computer simulation methods. A convenient formula can be borrowed from thermodynamics:

$$
P(E)=\exp \left(-\frac{E}{k T}\right)
$$

which gives the annealing probability $P(E)$ of a change on energy $E$ at temperature $T$, where $k$ is Boltzmann's constant.

Combining SA with MDS algorithm for localization process in a wireless ad hoc network, optimation procedure can be applied to each single node. Or in other words, for a given temperature $T$, all nodes in the path are considered one at a time. A random movement is generated by adding 


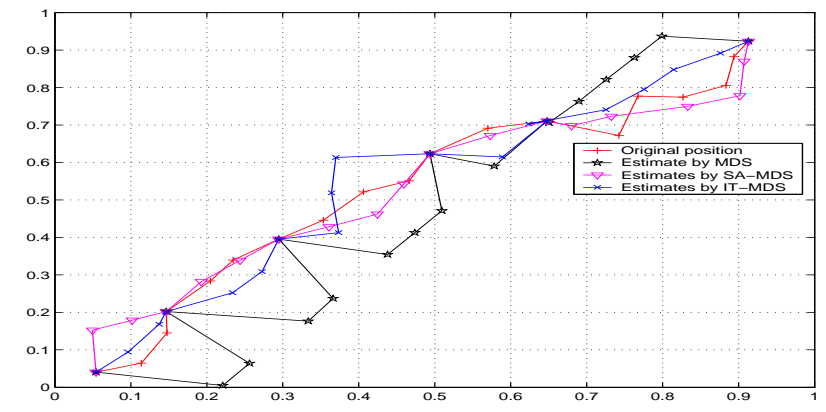

Figure 3. SA-MDS and IT-MDS algorithm example

a deviate from the Cauchy distribution to each coordinates $(1,2, \ldots, p)$ of node's position $X_{i}$ :

$$
x_{i k}=x_{i k}+T \cdot \tan (P), k=1,2, \ldots, p
$$

The cooling schedule for the temperature $T$ can use a simple method [29] as:

$$
T_{i+1}=a \cdot T_{i}, a \in(0,1)
$$

Consequently, the SA-MDS algorithm can be detailed below:

1) Define the temperature, $T_{\max }$ for highest temperature and $T_{\min }$ for the cooling down value;

2) Calculate the annealing probability from equation 4;

3) Update the displacement for nodes (one at a time) along the path using eqation 5;

4) Apply classical MDS algorithm to calculate the fitness function (equation 2): if a lower fitness function is obtained, keep the displacement from the above step; else, keep the displacement with certain probability;

5) update the temperature $T$ by equation 6 , and repeat steps $2,3,4$, and 5 until $T<T_{\min }$ or specified fitness function can be achieved.

A SA-MDS example (with IT-MDS) is given in Figure 3. The figure shows a path in a network of unit square area. ' $\nabla$ ' represents the estimated positions by SA-MDS method, and ' $X$ ' shows the estimates from IT-MDS. The true position is denoted by ' + '. The figure shows that the SA-MDS may achieve similar or even better estimates than the ITMDS method.

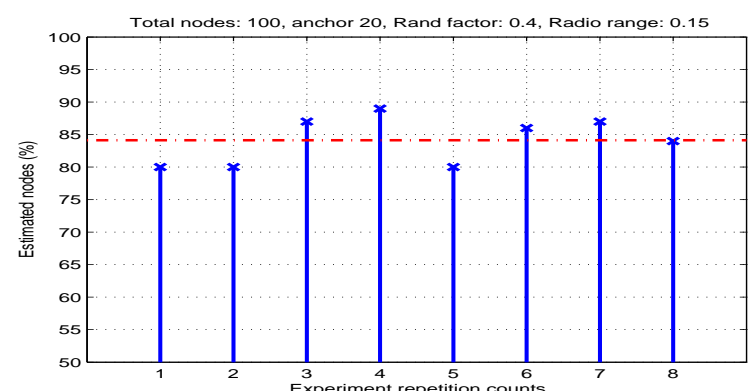

Figure 4. MDS estimation coverage analysis

\subsection{MDS Processing Coverage Analysis}

During the study, we found that the MDS algorithm can only estimate the position for a subset of the nodes directly in network. The reason lies in the fact: 1) path requirements ( $>3$ anchors) for the MDS algorithm, and 2) the DV-Hop propagation method that is used to minimize the traffic, it only selects the shortest path when routing.

A typical example is given in Figure 4, where a set of repetition experiments is carried out for a network of 100 nodes (20 anchors). The $\mathrm{x}$-axis denotes the experiment repetition, and the y-axis is the estimated nodes in percentage. The horizontal dotted line is the average estimation coverage. The results show the MDS algorithm can only estimate about $84 \%$ nodes in the network. Generally, if we enlarge the radio transmission range, then less nodes will get estimated. This necessities two remedies:

1. Another algorithm to estimate positions for the rest nodes in the network. In this research, we choose lateration algorithm to estimate the positions for the rest nodes.

2. Anchor deployment strategy. Similar to the research from Savarese [9], Doherty [14], and Nagpal [23], we manually deploy four anchor nodes in the corner of the unit square testing area.

\subsection{MDS Precision Analysis}

In this section, we consider the position estimation performance under a simple range measurement error model.

Sibson [32] considered the effect of perturbing the distance matrix $\Delta$ to a matrix $\Delta(\epsilon)$, such that $\Delta(\epsilon)=\Delta+$ $\epsilon F+O\left(\epsilon^{2}\right)$, where $F$ is a symmetric zero-diagonal matrix. Sibson showed that the corresponding induced eigenvalue $\lambda$ and the eigen vector $V$ will be:

$$
\begin{aligned}
& \lambda_{i}(\epsilon)=\lambda_{i}+\epsilon \mu_{i}+O\left(\epsilon^{2}\right) \\
& v_{i}(\epsilon)=v_{i}+\epsilon f_{i}+O\left(\epsilon^{2}\right)
\end{aligned}
$$


where $\mu_{i}=-\frac{1}{2} v_{i}^{T} F v_{i}, f_{i}=\frac{1}{2}\left(B-\lambda_{i} I\right)^{+} F v_{i}+$ $\frac{1}{2}\left(\lambda_{i} n\right)^{-1}\left(e^{T} F v_{i}\right) e$.

The superscript symbol ' + ' denotes pseudo-inverse, that is for a symmetric matrix $M$, with spectral decomposition $\sum \lambda_{k} v_{k} v_{k}^{T}$, then $M^{+}$is the matrix $\sum \lambda_{k}^{-1} v_{k} v_{k}^{T}: \lambda_{k} \neq 0$.

Consider a simple perturbation model $F$ where there is a constant off the diagonal and zero on it: $F=\epsilon\left(e e^{T}-I\right)$. From [32], we can get $\lambda_{i}(\epsilon)=\lambda_{i}+\frac{1}{2} \epsilon$.

Substitute $F$ to equation 8 , it is trival to derive the eigen vector $v_{i}(\epsilon)=v_{i}{ }^{1}$.

Consequently, the perturbation on the coordinate $X$ will be:

$$
x_{i}(\epsilon)-x_{i}=\left(\lambda_{i}+\frac{1}{2} \epsilon\right)^{\frac{1}{2}} v_{i}-\lambda_{i}^{\frac{1}{2}} v_{i}
$$

According to the binomial expansion, we can simplify the coordination perturbation as:

$$
\begin{aligned}
x_{i}(\epsilon)-x_{i} & =\left(\lambda_{i}+\frac{1}{2} \epsilon\right)^{\frac{1}{2}} v_{i}-\lambda_{i}^{\frac{1}{2}} v_{i} \\
& =\lambda^{\frac{1}{2}} v_{i}+\frac{1}{2} \lambda^{-\frac{1}{2}}\left(\frac{1}{2} \epsilon\right) v_{i}+\cdots+\left(\frac{1}{2} \epsilon\right)^{\frac{1}{2}} v_{i}-\lambda_{i}^{\frac{1}{2}} v_{i} \\
& =\frac{1}{2} \lambda^{-\frac{1}{2}}\left(\frac{1}{2} \epsilon\right) v_{i}+\cdots+\left(\frac{1}{2} \epsilon\right)^{\frac{1}{2}} v_{i} \\
& \simeq\left(\frac{1}{2} \epsilon\right)^{\frac{1}{2}} v_{i}+O(\epsilon)
\end{aligned}
$$

Formula 10 gives the position displacement of a node under constant distance perturbation. The result shows that the constant distance perturbation will not directly alter the directons (eigen vectors) of the deployment configuration, however, it will change the amount of the stretch for those directions (eigen values). In other words, the difference of the position coordinates is determined totally by the alteration of the eigen values.

\section{Experiments}

\subsection{Environment and Settings}

The simulation is built in Matlab (version 6.1). The main purpose for the experiments is to evaluate the performance of the proposed algorithms. We measure the estimation errors (see section 2.2) under various network settings. To cope with the ad hoc nature of the network, we run each test case ten times with the same network settings (not necessary the same network), and take the average values as the final results.

For the simulated annealing MDS (SA-MDS) algorithm, we select the initial temperature $T_{\max }$ value as the half radio range (i.e. $T_{\max }=\frac{R}{2}$ ). And for all simulation runs,

\footnotetext{
${ }^{1}$ Proof: $f_{i}=\frac{1}{2}\left(B-\lambda_{i} I\right)^{+} F v_{i}+\frac{1}{2}\left(\lambda_{i} n\right)^{-1}\left(e^{T} F v_{i}\right) e=\frac{1}{2} \epsilon(B-$ $\left.\lambda_{i} I\right)^{+} v_{i}+\frac{1}{2}\left(\lambda_{i} n\right)^{-1}\left(e^{T}\left(-\epsilon v_{i}\right)=0\right.$.
}
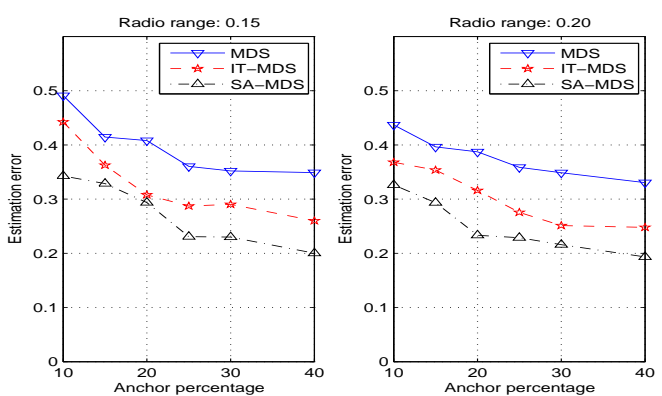

\section{Figure 5. Precision with the anchors (random- ness: 0.4)}

we use a network of 100 randomly deployed nodes in a unit square area. In addition, the network can update the following parameters:

- Anchor node density;

- Radio transmission range;

- Deployment randomness;

- Range measurement errors.

In the following sections, we first present the simulation results for MDS, IT-MDS and SA-MDS algorithms under various network conditions. Then, we outline the reported localization performance from other research groups. And finally, we compare, under the same network settings, the performance of proposed IT-MDS and SA-MDS algorithms with other representative algorithms.

\subsection{Simulation results for MDS, IT-MDS \& SA- MDS}

To understand the dependency of the estimation precision on the anchor population, we run the IT-MDS algorithm and the SA-MDS algorithm with different number of anchors in a set of networks with same network parameters. Figure 5 shows the relation between the precision and the anchor population. The $\mathrm{x}$-axis of the figure represents anchor percentage, and the $y$-axis denotes the estimation errors in term of radio transmission range.

The left chart of Figure 5 shows the results of radio range of 0.15 , and the right chart shows that of radio range of 0.2 . Both results indicate a trend that more anchors will lead a better performance.

Figure 5 also shows the relation between the estimation error and the radio transmission range. That is: with larger radio transmission range, both algorithms will give more accurate estimates. 


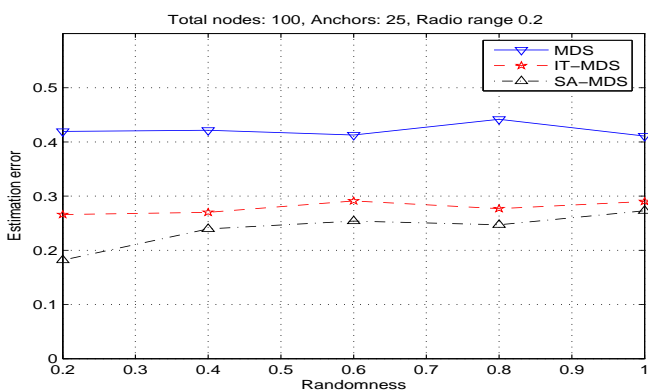

Figure 6. Precision with randomness

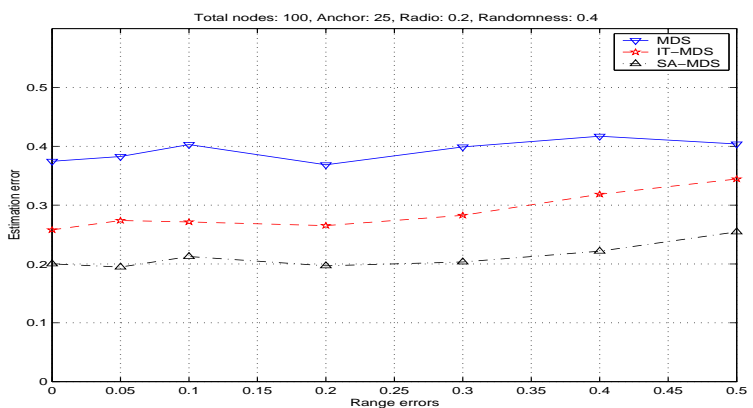

Figure 7. Precision with the 1-Hop range error

Figure 6 is the experimental results of the deployment randomness (x-axis) on the performance of the positioning precision (y-axis). It shows MDS based algorithms are insensitive to the deployment randomness.

To determine the effect of the 1-hop range measurement error of $\mathrm{DV}$-Hop method to the estimation precision, we simulate the 1-hop range error for both algorithms. The range error is determined relatively to the radio range; and to simulate the real situation, we define a maximum variance for each range measurement. The actual range error is determined dynamically during the experiment by the production of the maximum variance and a rand number between -1 and 1 .

The results are given in Figure 7. It shows that the estimation performance does not significantly decrease with the increasing 1-hop range errors. The main reason may because of the incorporation of the communication constraints during the iteration. Also we relocate all anchors' positions and adjust the corresponding distance among them after each estimation iteration. These procedures can potentially minimize the range errors.

To measure the accumulated errors for the Euclidean propagation method, we conducted similar experiments as for $D V$-Hop. The results is given in Figure 8 . The figure show the increasing estimation errors when the accumulated

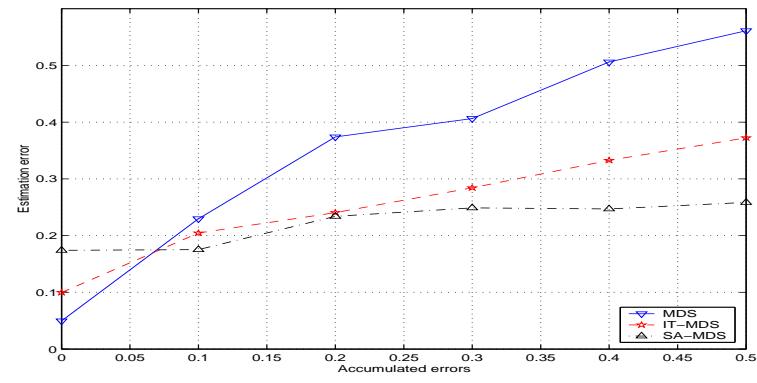

Figure 8. Precision with the accumulated Euclidean error

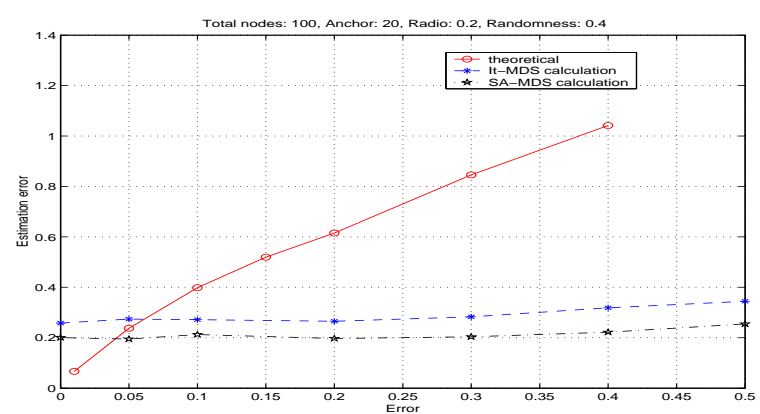

Figure 9. Precision analysis

errors increase.

Figure 9 shows the theoretical analysis for the MDS precision under constant perturbation (x-axis). It also gives the estimation results for IT-MDS and SA-MDS algorithms. Because our algorithms use $D V-H O P$ propagation method, the constant perturbation only affects the 1-hop pair-wise distance, with lower perturbation error, the estimation errors of calculation are higher than the theoretical results. However, the algorithms are fairly robust on the 1-hop pair-wise distance errors with only a little increasing of the estimation errors, this is contrast with the almost linear relation of the theoretical results.

\subsection{Algorithm Comparison}

\subsubsection{Reported performance from other research groups}

In order to evaluate the localization performance of the proposed algorithms, in this section, we first illustrate the reported localization performance from other research groups.

The results are given in Table 1. It can be seen that the location estimation performance is closely related with special network settings. Consequently, it is very difficult to 
Table 1. Reported estimation error in literature

\begin{tabular}{|l|l|l|}
\hline Algorithms & Condition \& settings & Estimation error \\
\hline \hline Probability algorithm & & \\
by Ramadurai and Sichitiu [26] & transmission range: 20m & $47 \%$ \\
\hline $\begin{array}{l}\text { Ad hoc positioning system } \\
\text { by Niculescu and Nath [15, 33] }\end{array}$ & $\begin{array}{l}\text { isotropic topology, 10\% anchor } \\
\text { isotropic topology, 20\% anchor }\end{array}$ & $35 \%$ \\
& anisotropic topology, 10\% anchors & $25 \%$ \\
& anisotropic topology, 20\% anchors & $900 \%$ \\
\hline Robust algorithm by Savarese [9] & connectivity > 7; anchor density $>5 \%$ & $33 \%$ \\
\hline $\begin{array}{l}\text { Approximate point-in-triangulation } \\
\text { test (APIT) algorithm by He [25] }\end{array}$ & higher power transmitters at anchors & $45 \sim 50 \%$ \\
\hline Amorphous algorithm by Nagpal [23] & local neighborhood density about 20 & $20 \sim 37 \%$ \\
\hline
\end{tabular}

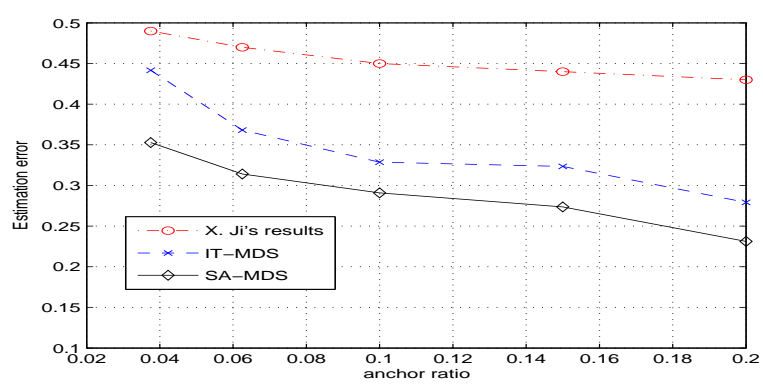

Figure 10. Comparison with X. Ji's iterative algorithm

compare them only with these values. On the other hand, it can be seen that the best reported estimation error is within $20 \% \sim 50 \%$ for networks with normal (isotropic) topology.

\subsubsection{Comparison under same network environment}

In this section, we first compare our IT-MDS and SA-MDS with the iterative MDS algorithm by X. Ji in [11]. Then we compare the localization performance of the proposed algorithms with three other representative algorithms under same networks of different deployment randomness.

Figure 10 gives the comparison results of our algorithms with the iterative algorithm by X. Ji. The test environment includes 400 nodes in a unit square area, the distance measurement error is 0.5 radio range. The $\mathrm{x}$-axis denotes the anchor ratio, and $y$-axis is the estimation error. X. Ji's estimation results is adopted directly from [11]. The simulation results show that the localization algorithms proposed in this paper give better estimation performance.

Figure 11 compares various localization algorithms under network environments with different randomness (see section 3.2). The $\mathrm{x}$-axis denotes the network randomness,

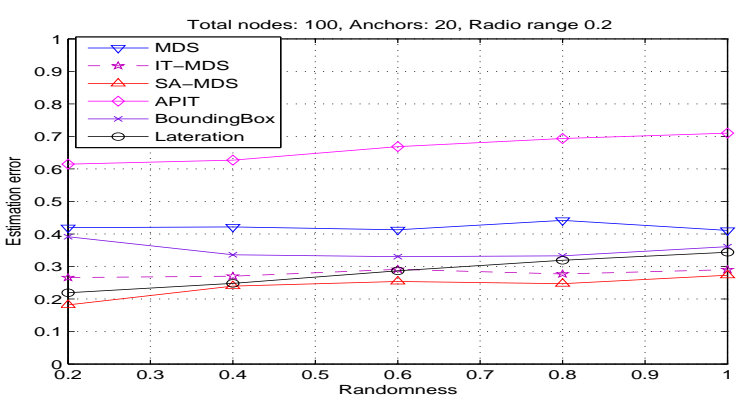

Figure 11. Comparison with other localization algorithms

and $y$-axis is the estimation error. The selected Grid overlaying method is the APIT method by He et al [25], and the bounding box method is by Savvides et al [10].

The test network contains 100 nodes, and anchor ratio is $20 \%$, radio transmission is 0.2 . In this experiment, we use $D V$-Hop radio propagation method to estimate the distance between communicating nodes. (The setting in this experiment is different from the original testing environment of the APIT algorithm (by He [25]) where higher power transmitter is used at anchors, so the estimation performance for this APIT algorithm is a little worse.)

From Figure 11, we can find that the SA-MDS algorithm performs better than all other algorithms, and the IT-MDS also achieves better estimation performance at larger deployment randomness.

For the test run (20\% anchors), we find that SA-MDS and IT-MDS algorithms present 18\% 25\% and 25\% 30\% estimation error, respectively, independent of the deployment randomness. Compare with the reported localization performance in literature (see Table 1), the proposed localization algorithms in this paper do yield better estimation 
performance.

\section{6 conclusion}

We proposed two algorithms, IT-MDS and SA-MDS, to enable nodes in a wireless network to estimate their positions. Both methods are based on a combination of multidimensional scaling method and lateration method. Specifically, IT-MDS algorithm considers the radio communication constraints, and adjust node's positions during each iteration; SA-MDS algorithm, on the other hand, mimicks the way of a metal cooling down procedure, and optimally locates the nodes in their most likely positions.

If $\mathrm{DV}$-Hop is used, both algorithms are insensitive to the 1-hop range measurement errors. With extensive simulation runs, results show that both algorithms provide accurate and consistent estimates no matter how precise each single estimate is. The average estimation errors are roughly bounded within $40 \%$ if a network contains more than $10 \%$ anchors and 0.2 radio range is used.

For future research, a theorital analysis of the impact of network parameters on the performance of the localization algorithms will be important

\section{References}

[1] T. Yan, T. He, and J. Stankovic, "Differentiated surveillance service for sensor networks," in Proceeding of First ACM Conference on Embedded Networked Sensor Systems (SenSys 2003), Los Angeles, CA, 2003.

[2] E. Kaasinen, "User needs for location-aware mobile services," Personal Ubiquitous Computing, vol. 7, no. 1, pp. 70-79, 2003.

[3] A. Cerpa, J. Elson, D. Estrin, L. Girod, M. Hamilton, and J. Zhao, "Habitat monitoring: application driver for wireless communications technology," in Proceedings of the ACM SIGCOMM Workshop on Data Communications, 2001, pp. 20-41.

[4] H. Wang, J. Elson, L.Girod, D. Estrin, and K. Yao, "Target classification and localizaiton in habitat monitoring," in Proceedings of the IEEE ICASSP 2003, Hong Kong, April 2003.

[5] E. Howden, "Networked sensors for the objective force," in Proceedings of SPIE 47th Annual Meeting, 2002.

[6] R.L.Moses, D. Krishnamurthy, and R. Patterson, "An auto-calibration method for unattended ground sensors," in Proc. ICASSP, May 2002, pp. 2941-2944.
[7] M. Perlman and Z. Haas, "Determining the optimal configuration for the zone routing protocol," IEEE Journal on Selected Areas in Communications, vol. 17, no. 8, pp. 1395-1414, Aug. 1999.

[8] Y. Ko and N. Vaidya, "Geotora: A protocol for geocasting in mobile ad hoc networks," Proceedings of IEEE ICNP 2000, pp. 240-250, Nov. 2000.

[9] C. Savarese, K. Langendoen, and J. Rabaey, "Robust positioning algorithms for distributed ad-hoc wireless sensor networks," Proceedings of the General Track: 2002 USENIX Annual Technical Conference, pp. 317327, 2002.

[10] A. Savvides, H. Park, and M. B. Srivastava, "The n-hop multilateration primitive for node localization problems," Journal of Mobile Networks and Applications (MONET), vol. 8, pp. 443-451, 2003.

[11] X. Ji and H. Zha, "Sensor positioning in wireless adhoc sensor networks using multidimensional scaling," in IEEE INFOCOM, 2004.

[12] K. Premaratne, J. Zhang, and M. Doguel, "Location Information-Aided Task-Oriented Self-Organization of Ad-Hoc Sensor Systems," IEEE sensors Journal, vol. 4, no. 1, Feb. 2004.

[13] L. Hu and D. Evans, "Localization for Mobile Sensor Networks," in MobiCom'04. ACM, Sept 2004.

[14] L. Doherty, K. S. J. Pister, and L. E. Ghaoui, "Convex position estimation in wireless sensor netowrks," Proc. of IEEE Inforcom, vol. 3, pp. 1655-1663, 2001.

[15] D. Niculescu and B. Nath, "Ad-hoc positioning system (APS)," in GLOBECOM 2001, San Antonio, Nov. 2001 .

[16] G. Young and A. Householder, "Discussion of a set of points in terms of their mutual distances," Psychometrika, vol. 3, pp. 19-22, 1938.

[17] T. F. Cox and M. A. Cox, Multidimensional Scaling, 2nd ed. Chapman and HallCRC, 2001.

[18] D. Niculescu and B. Nath, Eds., Ad Hoc Positioning System (APS) using AoA. San Francisco, CA: IEEE INFOCOM, 2003.

[19] C. Savarese, "Robust positioning algorithms for distributed ad-hoc wireless sensor networks," Master's thesis, Berkeley, 2002.

[20] A. Savvides, H. Park, and M. Srivastava, "The bits and flops of the n-hop multilateration primitive for node 
location problems," First ACM International Workshop on Wireless Sensor Networks and Application (WSNA), pp. 112-121, 2002.

[21] K. Kleinrock and J. Silvester, "Optimum tranmission radii for packet radio networks or why six is a magic number," in in National Telecommunications Conference, Birmingham, Alabama: IEEE, Dec. 1978, pp. 4.3.1-4.3.5.

[22] R. Nagpal, "Organizing a global coordinate system from local information on an amorphous computer," A.I. Memo 1666, Aug. 1999.

[23] R. Nagpal, H. Shrobe, and J. Bachrach, "Organizing a global coordinate system from local information on an ad hoc sensor network," In the 2nd International Workshop on Information Processing in Sensor Networks (IPSN’03), April 2003.

[24] A. Savarese, J. Rabaey, and J. Beutel, "Locationing in distributed ad-hoc wireless sensor networks," In IEEE International Conference on Acoustics, Speech and Signal Processing (ICASSP), pp. 2037-2040, May 2001.

[25] T. He, C. Huang, B. M. Blum, J. A. Stankovic, and T. Abdelzaher, "Range-free location schemes for large scale sensor networks," MobiCom'03, Sep. 2003.

[26] V. Ramadurai and M. L. Sichitiu, "Localization in wireless sensor networks: A probabilistic approach," in Proc. of the 2003 International Conference on Wireless Networks (ICWN 2003), Las Vegas, NV, June 2003, pp. 275-281.

[27] M. L. Sichitiu, V. Ramadurai, and P. Peddabchagari, "Simple algorithm for outdoor localization of wireless sensor networks with inaccurate range measurements," in Proceedings of the International Conference of Wireless Networks. Las Vegas, Nevada, USA: CSREA Press 2003, June 2003.

[28] N. Metropolis, A. Rosenbluth, M. N. Rosenbluth, A. Teller, and E. Teller, "Equations of state calculations by fast computing machines," J. Chem. Phys., vol. 21, pp. 1087-1092, 1958.

[29] K. Dowsland, "Simulated annealing," in Modern Heuristic Techniques for Combinatorial Problems, chapter 2. McGraw-Hill Book Company, Berkshire, C. R. Reeves, Ed., 1995.

[30] M. Picus, "A monte carlo method for the approximate solution of certain types of constrained optimization problems," Oper. Res. 18, pp. 1225-1228, 1970.
[31] S. Kirkpatrick, C. J. Gerlatt, and M. P. Vecchi, “Optimization by simulated annealing," Science, vol. 200, pp. 671-680, 1983.

[32] R. Sibson, "Stuides in the Robustness of Multidimensional Scaling: Perturbational Analysis Classical Scaling," Journal of the Royal Statistical Society. Series B (Methodological), vol. 41, no. 2, pp. 217-229, 1979.

[33] D. Niculescu and B. Nath, "Localized positioning in ad hoc networks," IEEE SNPA 2003, May 2003. 